\title{
First Real-Time 100-Gb/s NRZ-OOK Transmission over 2 km with a Silicon Photonic Electro-Absorption Modulator
}

\author{
J. Verbist ${ }^{1,2, *}$, M. Verplaetse ${ }^{1}$, S.A. Srivinasan ${ }^{2,4}$, P. De Heyn ${ }^{4}$, T. De Keulenaer ${ }^{1,3}$, R. Pierco ${ }^{1,3}$, R. \\ Vaernewyck $^{1,3}$, A. Vyncke ${ }^{1,3}$, G. Torfs ${ }^{1}$, X. Yin ${ }^{1}$, G. Roelkens ${ }^{2}$, J. Van Campenhout ${ }^{4}$, , J. Bauwelinck ${ }^{1}$ \\ ${ }^{1}$ IDLab, INTEC, Ghent University - imec, 9052 Ghent, Belgium \\ ${ }^{2}$ Photonics Research Group, INTEC, Ghent University - imec, 9052 Ghent, Belgium \\ ${ }^{3}$ BiFast, Spin-off incubation project of Ghent University-imec, IDLab, 9052 Ghent, Belgium \\ ${ }^{4}$ imec, Kapeldreef 75, 3001 Leuven, Belgium \\ *E-mail: jochem.verbist@ugent.be
}

\begin{abstract}
We demonstrate the first real-time, serial $100 \mathrm{~Gb} / \mathrm{s}$ NRZ-OOK transmission with an integrated GeSi EAM implemented on a silicon photonics platform. Transmission over 500m of SSMF and $2 \mathrm{~km}$ of dispersion shifted fiber is presented.

OCIS codes: (250.5300) Photonic Integrated Circuits, (200.4650) Optical Interconnects; (060.4080) Modulation
\end{abstract}

\section{Introduction}

In order to meet the growing bandwidth requirements, data centers push the research towards short-reach optical interconnects operating at $100 \mathrm{~Gb} / \mathrm{s}$ and beyond. Currently the evolution from $100 \mathrm{~Gb} / \mathrm{s}$ Ethernet to $400 \mathrm{~Gb} / \mathrm{s}$ transceivers is under discussion within the IEEE P802.3bs 400 Gigabit Ethernet Task Force[1]. Several implementation options including multi-lane, multiple polarizations and/or multi-level transmissions are under investigation. A four lane $100 \mathrm{~Gb} / \mathrm{s} \mathrm{NRZ} \mathrm{scheme} \mathrm{could} \mathrm{provide} \mathrm{an} \mathrm{elegant} \mathrm{solution} \mathrm{to} \mathrm{achieve} 400 \mathrm{GbE}$ transceivers allowing higher spatial efficiency through lower lane counts, while maintaining the low complexity of OOK-based, low-power electronics. Silicon photonics is ideally suited to provide compact and low-cost transceivers for optical interconnects, although scaling to $100 \mathrm{~Gb} / \mathrm{s}$ lane rates has proven to be difficult for silicon-based transceivers. Recently, $100 \mathrm{~Gb} / \mathrm{s}$ single-lane links have been realized using four level pulse amplitude modulation (PAM-4), discrete multi-tone (DMT) and electrical duobinary (EDB) [2-5]. However, these experiments rely on heavy and powerconsuming digital signal processing (DSP), typically done off-line. Examples of true real-time $100 \mathrm{~Gb} / \mathrm{s}$ transmissions without DSP are limited. In [6], $112 \mathrm{~Gb} / \mathrm{s}$ PAM-4 modulation on a discrete Mach-Zehnder Modulator was demonstrated at $8.6 \mathrm{~W}$ of power consumption. EDB was used to achieve real-time $100 \mathrm{~Gb} / \mathrm{s}$ on a InP-based travellingwave electro-absorption with integrated DFB-laser [7]. The transmission line structure of the modulator electrodes necessitates a power consuming $50 \Omega$ termination. In [9], an impressive real-time BER lower than $10^{-10}$ was achieved with silicon-organic Mach-Zehnder modulator, again with travelling wave electrodes and $50 \Omega$ termination. . However, this device does have several important drawbacks in terms of cost, power and footprint, when envisioned as a shortreach optical interconnect transceiver. The $1.1 \mathrm{~cm}$ long modulator was packaged with two InP-based electrical multiplexers offering a differential voltage swing of $6 \mathrm{Vp}$, resulting in a power consumption -excluding the laser-of almost $6.85 \mathrm{~W}$. The transmitter module measures $6.5 \times 2 \mathrm{~cm}$. The proposed $4 \times 100$ PIN-DEMUX receiver adds another $5.5 \mathrm{~W}$ of power consumption and measures $4 \times 6.9 \mathrm{~cm}$. Due to the high insertion loss of the transmitter the maximal optical power was limited to $-9.5 \mathrm{dBm}$, making an EDFA-less link even with the addition of a high-speed transimpedance amplifier difficult. While fabricated on a silicon platform, the organic modulator still requires postprocessing to deposit the organic material.

In this paper we present a real-time, serial and single-wavelength $100 \mathrm{~Gb} / \mathrm{s}$ NRZ-OOK link based on an extremely compact Germanium-silicon electro-absorption modulator integrated on a $200 \mathrm{~mm}$ silicon platform in combination with in-house designed transmitter (TX IC) and receiver (RX IC) chipsets in a SiGe BiCMOS technology. The modulator was driven lumped with $2 \mathrm{Vpp}$ without any termination. This is the first real-time, single channel $100 \mathrm{~Gb} / \mathrm{s}$ NRZ transmission on a silicon-based integrated modulator without any need for material post-processing or complex DSP. Transmission over $500 \mathrm{~m}$ of standard single mode fiber (SSMF) and $2 \mathrm{~km}$ of dispersion shifted fiber (DSF) is reported.

\section{Experiment Setup}

The GeSi EAM consists of a 600nm wide and $80 \mu \mathrm{m}$ long germanium waveguide with embedded lateral p-i-njunction, connected via tapers to silicon waveguides, and was fabricated on imec's $200 \mathrm{~mm}$ silicon photonics platform. Modulation is obtained through the Franz-Keldish effect, which shifts the bandgap edge of GeSi by applying an electrical field [9]. Light is coupled in and out of the waveguide device by fiber-to-chip grating couplers which have 
an insertion loss of $\sim 6 \mathrm{~dB} /$ coupler. An electrical RF probe (without $50 \Omega$ termination) is used to apply the bias voltage and high speed signal.
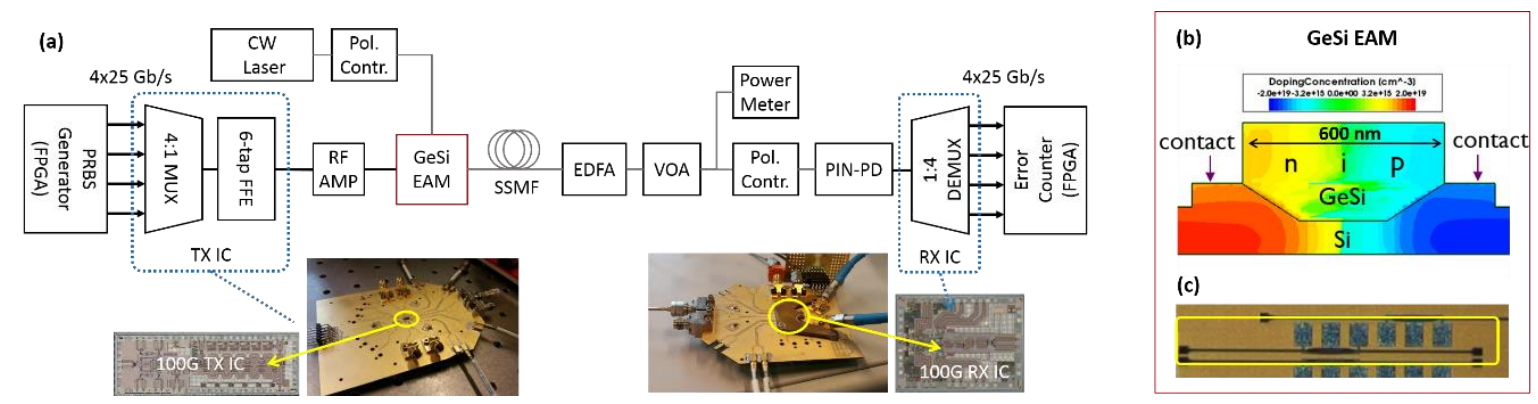

Fig. 1. (a) Experiment setup of real-time $100 \mathrm{~Gb} / \mathrm{s}$ NRZ-OOK optical link, (b) cross-section of the GeSi waveguide EAM; (c) micrograph of the presented $80 \mu \mathrm{m}$ modulator

The experiment setup is shown in Fig.1. A XilinX VirteX FPGA generates four $2^{7}-1$ long pseudo-random bit streams (PRBS) at $25 \mathrm{~Gb} / \mathrm{s}$, which are multiplexed with the required delays to form again a $2^{7}-1$ long PRBS at $100 \mathrm{~Gb} / \mathrm{s}$.

A six-tap analog feedforward equalizer (FFE) on the transmitter IC (TX IC) is set to compensate the frequency roll-off and non-idealities of the following components and the link. A $50 \mathrm{GHz} \mathrm{RF}$ amplifier with internal bias-T delivers the pre-emphasized electrical signal with a $2 \mathrm{Vpp}$ swing to the optical modulator. The in-waveguide laser power was $\sim 5 \mathrm{dBm}$ at a wavelength of $1601.5 \mathrm{~nm}$. The EAM was biased at $-1.85 \mathrm{~V}$ for back-to-back experiments and at $-2.05 \mathrm{~V}$ for transmission experiments, resulting in a photocurrent of roughly $3.60 \mathrm{~mA}$ and $3.80 \mathrm{~mA}$, respectively. The fiber-coupled power after the photonic IC was almost $-5 \mathrm{dBm}$. With these settings we measured a dynamic extinction ratio of $\sim 6 \mathrm{~dB}$ and an insertion loss around $\sim 6 \mathrm{~dB}$ for the modulator. To convert the optical signal back to the electrical domain, a commercial $50 \mathrm{GHz}$ PIN-photodiode with a responsivity near $0.6 \mathrm{~A} / \mathrm{W}$ was used. As no highspeed $(>50 \mathrm{GHz})$ transimpedance amplifier was available, an EDFA and variable optical attenuator were used to increase and control the signal power before coupling the light into the PIN-PD. The EDFA can be removed from the setup by addition of a transimpedance amplifier, which as an added benefit would also improve the transceiver performance by reducing the amount of noise in the O/E/O-link.

Finally, the $100 \mathrm{~Gb} / \mathrm{s}$ data is deserialized by the electrical receiver (RX IC) into 4 x $25 \mathrm{~Gb} / \mathrm{s}$ streams and fed back to the same FPGA for real-time error detection without any complex DSP or offline processing. The RX IC consists of 2 parallel level-shifting limiting amplifiers (LA), an XOR-gate and a 1-to-4 DEMUX, allowing operation as an EDB decoder (using both LAs) or as a NRZ decoder (keeping the output of one LA fixed). Both transmitter and the receiver IC were designed in-house in a $0.13 \mu \mathrm{m}$ BiCMOS technology [10]. The RX-IC occupies $\sim 1950$ x $2600 \mu \mathrm{m}$ and consumes less than $1.2 \mathrm{~W}$, the TX IC occupies $\sim 1500 \times 4500 \mu \mathrm{m}$ and consumes $1 \mathrm{~W}$.

\section{Results and Discussion}

Real-time BER test were carried out to characterize the optical link performance with the setup shown in Fig. 1. For back-to-back (B2B) transmission a BER below $6 \times 10^{-9}$ was obtained for an average optical input power to the PIN$\mathrm{PD}$ of $8.3 \mathrm{dBm}$. The hard-decision forward error coding limit (BER $=3.8 \times 10^{-3}$ for a $7 \%$ overhead) is reached for an average power of $-0.6 \mathrm{dBm}$. Compared to the fiber-coupled power after the modulator, this requires an increase of only $\sim 4.5 \mathrm{~dB}$ which could be realized by replacing the on-chip grating couplers ( $\sim 6 \mathrm{~dB} /$ coupler) by low-loss edgecouplers $(<2 \mathrm{~dB} /$ coupler) [11]. This indicates that an EDFA-less link is indeed possible.

The frequency response of the optical link for different fiber lengths is shown in Fig. 2(e). Operation in L-band $(1601.5 \mathrm{~nm})$ severely degrades the flatness of the frequency response for longer fiber spans due to high levels of chromatic distortion. Nevertheless, we still manage to obtain transmission over more than $500 \mathrm{~m}$ of SSMF with a BER down to $2 \times 10^{-5}$. Sub-FEC operation is achieved for powers $>1.5 \mathrm{dBm}$, resulting in a penalty of $2 \mathrm{~dB}$ with respect to B2B. Finally, transmission over $2 \mathrm{~km}$ of DSF ( $\sim \mathrm{ps} / \mathrm{nm} . \mathrm{km}$ dispersion) assuming FEC is also achieved for a received power of more than $4 \mathrm{dBm}$, saturating in an error-floor of $1 \times 10^{-3}$. Investigating the received eyes taken at the output of the PIN-PD in Fig. 2(a-c), we believe that the limited bandwidth of the RX IC (41 GHz) is the main contributor to the total BW, limiting the performance of the overall link. Tests with a new and faster RX IC are planned in the coming months. 

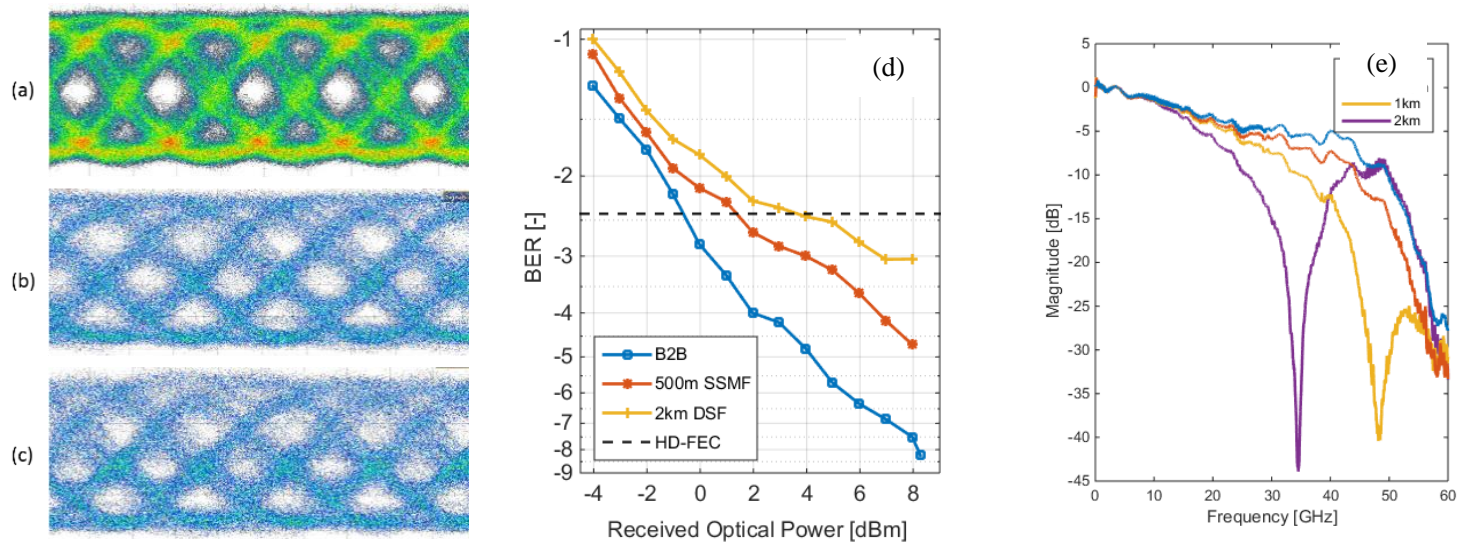

Fig. 2: Eye diagrams and for (a) $100 \mathrm{~Gb} / \mathrm{s} \mathrm{B} 2 \mathrm{~B}$, (b) $500 \mathrm{~m} \mathrm{SSMF}$ and (c) $2 \mathrm{~km}$ of DSF transmission captured after the PIN-PD by a $70 \mathrm{GHz}$ sampling scope corresponding to a received optical power of $6 \mathrm{dBm}$; (d) Real-time BER curves for the same fiber lengths;

(e) Frequency response of the optical link (from RF Amp to PIN-PD) for different lengths of SSMF at $1601.5 \mathrm{~nm}$

An additional advantage of the proposed modulator is that the same structure could be used as a high-speed photodiode. Fig. 3 shows an example of the received eye for such an EAM-to-EAM optical link at $100 \mathrm{~Gb} / \mathrm{s}$ where an identical copy of the EAM on a separate die was used as a receiver. The bias voltage for the RX EAM was applied through an electrical RF probe and set to $5 \mathrm{~V}$ to ensure maximal light absorption. Although this was not further investigated, comparing the received eye in Fig.3 to that from a commercial IIIV-based PIN-PD in Fig.2(a) we can see a small degradation for the EAM as PD. This is partially be attributed to the absence of a well-matched $50 \Omega$ output of the EAM-PD, which is expected by the RX IC and is internally present on the commercial PIN-PD. Nevertheless, a complete silicon transceiver with $100 \mathrm{~Gb} / \mathrm{s}$ NRZ-OOK line rates link based on a GeSi EAM as a modulator and photodetector is achievable.

(a)

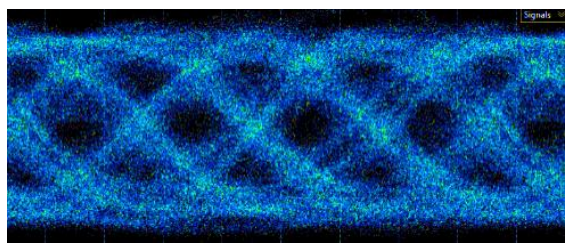

(b)

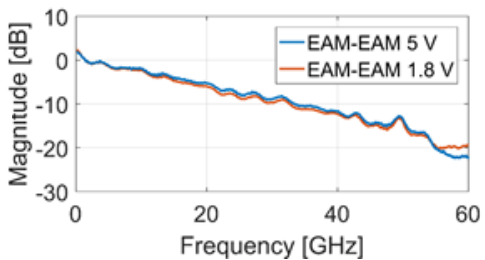

Fig. 3: (a) Eye diagram for a 100Gb/s EAM-to-EAM link (TX-EAM at 1.8V, PD-EAM at 5V); (b) Frequency response of the EAM-to-EAM link for different EAM-PD reverse bias voltages.

\section{Conclusion}

We have demonstrated a serial $100 \mathrm{~Gb} / \mathrm{s}$ NRZ-OOK transmission based on an integrated GeSi EAM, fabricated in a $200 \mathrm{~mm}$ silicon photonics platform in combination with in-house designed SiGe BiCMOS transmitter and receiver chipset. Successful transmission over 500m of SSMF and $2 \mathrm{~km}$ of DSF was achieved and verified in real-time without the need for any DSP. These results showcase the capabilities of silicon photonics as a possibly disruptive technology for compact and low-power transceivers for $400 \mathrm{GbE}$ short-reach optical interconnects.

\section{Acknowledgement}

This work was supported by the imec IAP on Optical I/O for the Ge EAM, the UGent IOF for the Tx/Rx ICs, the H2020 TERABOARD project, the UGent BOF and the FWO.

\section{References}

[1] "IEEE P802.3bs 400 Gigabit Ethernet Task Force." [Online]. Available: http://www.ieee802.org/3/bs/

[2] C. Caillaud et al., "Low cost $112 \mathrm{~Gb} / \mathrm{s}$...," in ECOC, Valencia, 2015, PDP

[3] P. Dong et al., "Four-channel 100-Gb/s...," in OFC, Los Angeles, CA, 2015, Th5B.4 - PDP

[4] W. Bo et al., "Single-Lane ...," in ECOC, Dusseldorf, Germany, 2016, pp. 1148-1150

[5] J. Lee et al., "Serial 103.125-Gb/s transmission...," in OFC, 2014, San Francisco, CA, 2014, PDP - Th2.A.4
[6] J. Lee et al, in ECOC , ID:0604, Valencia, 2015.

[7] X. Yin et al., "First Demonstration of ...," in ECOC,

Dusseldorf, Germany, 2016, PDP

[8] Katopodis et al. "Tunable transmitter ...", in OFC, Anaheim, CA, 2016, Tu2A3

[9] S. A. Srinivasan et al, OFC, Anaheim, CA, 2016, pp. 1-3. [10] M. Verplaetse et al., "Real-Time $100 \mathrm{~Gb} / \mathrm{s}$...," in Journ. Of Lightw. Techn., 2017, available online

[11] A. Dewanjee et al., "A low-loss...," 2014 IEEE Photonics Conference, San Diego, CA, 2014, pp 